\title{
Gregor Wedekind
}

\section{EINLEITUNG}

Der Sammelband Max Slevogts Netzwerke führt Bemühungen zur Erneuerung der Slevogtforschung fort, die seit einigen Jahren im Gange sind und die zwischenzeitlich zur Gründung des Max Slevogt-Forschungszentrums geführt haben, ein auf der Grundlage der Kooperation des Instituts für Kunstgeschichte und Musikwissenschaft der Johannes Gutenberg-Universität, des Landesmuseums Mainz und der Pfälzischen Landesbibliothek in Speyer bestehenden Zusammenschlusses von Forschern. Der 2016 erschienene Band Blick zurück nach vorn. Neue Forschungen zu Max Slevogt ${ }^{1}$ unternahm zunächst den Versuch, das Feld der Slevogtforschung im Moment eines doppelten Umbruchs abzustecken: Zum einen war 2015 mit Slevogts grafischem Nachlass das letzte große Konvolut seines Künstlernachlasses aus der Obhut der Familie in die öffentliche Hand übergegangen: Er ist nun im Besitz der Stiftung Rheinland-Pfalz für Kultur und wurde mit Unterstützung der Kulturstiftung der Länder sowie der Generaldirektion Kulturelles Erbe Rheinland-Pfalz erworben und befindet sich als Dauerleihgabe im Landesmuseum Mainz. Zum andern galt es, den sich abzeichnenden Generationenwechsel in der Slevogtforschung als eine besondere Herausforderung produktiv anzunehmen. Als dessen Zäsur kann die 2014 gezeigte Ausstellung Neue Wege des Impressionismus gelten, mit der Sigrun Paas so etwas wie eine Summe ihrer langjährigen Tätigkeit als Kustodin der Slevogtbestände des Landesmuseums Mainz vorlegte. ${ }^{2}$ Auf der anlässlich dieser Ausstellung vom Institut für Kunstgeschichte und Musikwissenschaft der Johannes Gutenberg-Universität im Zusammenwirken mit dem Landesmuseum Mainz ausgerichteten Tagung und der daraus resultierenden Publikation ging es zunächst darum, das Forschungsfeld neu zu vermessen bzw. erste Sondierungen vorzunehmen, wie es um dieses Forschungsfeld überhaupt bestellt ist. Damals wurde deutlich, dass für Slevogt wesentliche Zusammenhänge, die die Kunstkritik, die Sammlungsgeschichte, die Verortung seiner Person im Rahmen einer intellectual bistory des späten Kaiserreichs und der Weimarer Republik sowie ganz generell die kulturgeschichtliche Einordnung seines Schaffens betreffen, erst noch zu erarbeiten sind. Dieser Perspektive haben wir daher ein zweites wissenschaftliches Kolloquium gewidmet, das am 29. und 30. November 2018 vom Max Slevogt-Forschungszentrum ausgerichtet wurde. Die Beiträge dieses Kolloquiums versammelt der hier vorliegende Band.

1 Blick zurück nach vorn. Neue Forschungen zu Max Slevogt, hg. von Gregor Wedekind in Verbindung mit der Generaldirektion Kulturelles Erbe Rheinland-Pfalz, Berlin und Boston: De Gruyter, 2016 (Phoenix - Mainzer kunstwissenschaftliche Bibliothek, 2).

2 Max Slevogt. Neue Wege des Impressionismus, hg. von der Direktion des Landesmuseums Mainz, bearbeitet von Sigrun Paas, Kat. Ausst. Mainz, Landesmuseum, München: Hirmer, 2014. 
Wenn in den in ihm angestellten Überlegungen der Netzwerkbegriff eine Rolle spielt, dann deswegen, weil dieses Denkbild verspricht, ein Beschreibungsmodell für die überaus komplexe künstlerische Praxis Max Slevogts und ihrer geistigen, materiellen und kommunikativen Voraussetzungen abgeben zu können. ${ }^{3}$ Es gilt, die Knotenpunkte dieses Netzwerk mittels historischer Arbeit zu identifizieren, wobei wir es sind, die diese Knotenpunkte festlegen und sie verbinden. In gewisser Weise ist das Netzwerk somit eine Kippfigur. Max Slevogts Netzwerke auf der einen Seite, die in der konkreten Plastizität ihrer historischen Verästelungen für uns jedoch gar nicht mehr erreichbar sind, und - auf der anderen Seite - die kulturellen und künstlerischen Netzwerke, in denen wir Slevogts Person und seine Kunst verorten, als ein kognitives mapping, das, indem es immaterielle historische Netzwerkstrukturen sichtbar machen will, diese induziert und etabliert. Unsere Netzwerke also, nicht die Slevogts. Netze, die wir als projektive kunst- und kulturhistorische Netze über sein Werk werfen, um es einzufangen. Wir sind es, die ein Netz knüpfen, im Sinne einer Verknüpfung aller verfügbaren und auffindbaren Materialien, ob schriftlicher, visueller oder wie auch immer dinglicher Natur, die mit dem Werk Slevogts verbunden sind. Was im vorliegenden Band als jeweils punktuelle Forschung sich äußert, wollen wir in der Zukunft mit einem klassischen kunsthistorischen Instrumentarium verknüpfen, einem Werkkatalog oder auch einem Catalogue raisonné, der für Slevogt erstaunlicherweise immer noch fehlt.

Dabei sei betont, dass es bei der kunsthistorischen Netzwerkforschung nicht darum geht, Netzwerke etwa im Sinne einer bloßen historischen Rekonstruktion personeller Konstellationen aufzufinden, sondern es ihr Ziel ist, Informationen und Daten zu verknüpfen, um Aufschluss über das künstlerische Werk zu erlangen. Wie ja auch die Auswertung der Nachlassquellen im Wesentlichen daraufhin betrieben werden sollte, welchen Erkenntnisgewinn über die einzelnen künstlerischen Arbeiten und die Kunstproduktion im Ganzen, des Euvres, und dessen Einbettung in die Kunstgeschichte sie ermöglicht.

3 Zum Netzwerkbegriff siehe: Peter Kappelhoff, „Netzwerk“, in: Wörterbuch der Soziologie, hg. von Günter Endruweit und Gisela Trommshoff, 3 Bde., Stuttgart: Enke, 1989, Bd. 2, S. 465-467; Soziale Netzwerke. Konzepte und Methoden der sozialwissenschaftlichen Netzwerkforscbung, hg. von Johannes Weyer, München: Oldenbourg, 2000; Thomas Goschke, „Netzwerk“ in: Gedäcbtnis und Erinnerung. Ein interdiziplinäres Lexikon, hg. von Nicolas Pethes und Jens Ruchartz, Reinbek bei Hamburg: Rowohlt, 2001, S. 411-414; Mark Wigley, „Network Fever“, in: Grey Room, Bd. 4, 2001, S. 82-12; Christiane Heibach, „Netzwerke: Neue Visualisierungsstrategien zwischen Kunst und Wissenschaft" in: Bild Wissen Medien. Visuelle Kompetenz im Medienzeitalter, München: Kopaed, 2002, S. 287-302; Hartmut Böhme, „Netzwerke. Zur Theorie und Geschichte einer Konstruktion“, in: Netzwerke. Eine Kulturtechnik der Moderne, hg. von Jürgen Barkhoff, Hartmut Böhme und Jeanne Riou, Köln: Böhlau, 2004 (Literatur - Kultur - Geschlecht. Studien zur Literatur- und Kulturgeschichte, 29), S. 17-36; Netzwerke der Moderne. Erkundungen und Strategien, hg. von Jan Broch, Markus Rassiller und Daniel Scholl, Würzburg: Königshausen \& Neumann 2007; Julia Gelshorn und Tristan Weddigen, „Das Netzwerk. Zu einem Denkbild in Kunst und Wissenschaft“, in: Grammatik der Kunstgescbicbte. Sprachproblem und Regelwerk im 'Bild-Diskurs'. Oskar Bätscbmann zum 65. Geburtstag, hg. von Hubert Locher und Peter J. Schneemann, Zürich, Emsdetten und Berlin: Edition Imorde, 2008, S. 54-77; Vom Spinnen in der Kunst, hg. von Anette Hüsch, Kat. Ausst. Kiel, Kunsthalle, Bielefeld: Kerber, 2014. 
Wir stellen die Frage nach dem Netzwerk, um etwas über den Stellenwert von Ideen, Materialien, Techniken herauszufinden, aber auch über Horizonte der künstlerischen Position und Positionierung. Nicht länger soll Slevogt aus sich selbst heraus erklärt werden, er in Repetition des ewigen Künstlermythos als ein aus sich selbst schöpfender Künstler gesehen werden, dessen Werk sich dann dementsprechend immer nur als Entwicklung in tautologischen Kausalketten unter Zuhilfenahme von kunsthistorischen Denkfiktionen wie Stilwandel oder Stilabfolge erklärt werden kann. Auch im Bereich der Kunst handelt es sich um kollektive Formationen. Das künstlerische Werk ist nicht einfach das Resultat eines einsamen Entschlusses des Künstlers in seinem Atelier, sondern es hat einen kollektiven gesellschaftlichen Rahmen. Im Verhältnis zwischen den verschiedenen Akteuren im künstlerischen Feld werden bestimmte Annahmen und Interessen sichtbar. Worüber haben sie nachgedacht, worüber sich ausgetauscht, wie haben sie miteinander gearbeitet und interagiert: Dann kann man Rückschlüsse auf das Werk ziehen und damit das Werk verstehen als ein Beitrag innerhalb eines kulturellen Feldes. Das es zu kartieren gilt.

Allein schon wegen der Größe dieses Feldes kann der hier vorliegende Band in keiner Form beanspruchen, es auch nur annähernd abzudecken. Vielmehr hat man es ganz wesentlich mit bruchstückhaften Annäherungen und Anläufen zu tun. Geht man allein von jenen Personen aus, die für Slevogts Werdegang zu bestimmten Zeiten eine wichtige Rolle gespielt haben und für die er in manchen historischen Momenten und in gewissen Hinsichten eine wichtige Rolle spielte, kommt man zu einer langen Liste von Namen. Um nur die Bekanntesten zu nennen: Wilhelm Bode, Bruno und Paul Cassirer, Lovis Corinth, Bruno Eisner, Julius Elias, Julius Freund, Max J. Friedländer, Ernst Fuchs, Otto Gerstenberg, Curt Glaser, Johannes Guthmann, Richard Hamann, Gustav Hartlaub, Wilhelm Hausenstein, Emil Heilbut, Ludwig Justi, Heinrich Kohl sowie dessen Sohn Franz-Josef Kohl-Weigand, ${ }^{4}$ Alfred Kubin, Alfred Lichtwark, Max Liebermann, Julius Meier-Graefe, Emil Orlik, Max Osborn, Gustav Pauli, Walter Passarge, János Plesch, Hans Purrmann, Hans Rosenhagen, Karl Scheffler, Johannes Sievers, Carl Steinbarth, Hugo von Tschudi, Karl Voll, Emil Waldmann, Fritz Wichert oder Konrad Wrede. All diese Museumsleute, Publizisten, Galeristen, Künstlerkollegen, Kunstkritiker, Sammler und Freunde, Mitstreiter und Weggefährten gälte es in ihrem Verhältnis zu Slevogt eigens zu untersuchen. Doch nur einige wenige von ihnen konnten in unserem Band Berücksichtigung finden. Zudem haben wir eine sehr weite Auslegung des Netzwerkbegriffs zugrundegelegt, der nicht nur Bezüge zwischen Personen oder zu Institutionen, sondern auch zu materiellen Infrastruk-

4 Zu Kohl-Weigand erschien vor einigen Jahren ein Artikel von Kirsten Fitzke, „Franz Josef Kohl-Weigand: Ein Slevogt-Sammler schreibt Forschungsgeschichte“, in: Mobilitas. Festscbrift zum 70. Geburtstag Werner Schreiners, hg. von Klaus Frédéric Johannes, Neustadt an der Weinstraße 2017 (Schriftenreihe der Bezirksgruppe Neustadt an der Weinstraße im Historischen Verein der Pfalz, N.F.1), S. 547-566. Was ein wertvoller Beitrag im Bemühen der Erneuerung der Slevogtforschung hätte sein können, wird durch die Polemik der Autorin, die der Slevogtforschung pauschal Kritiklosigkeit gegenüber der älteren Forschung und Ignoranz wichtiger Archivquellen vorwirft, zu einer verzerrten und unproduktiven Selbstabgrenzung 
turen wie Farbenhandlungen oder Bibliotheken sowie Bezüge zwischen Bildern - den eigenen und denen anderer Künstler - umfasst. Immer noch geht es also um erste Ansätze und die sukzessive Erweiterung unserer Kenntnisse und unseres Verstehens.

Unsere Anläufe sind von anderen hinterfangen worden, hat doch der 150. Geburtstag des Künstlers 2018 dazu beigetragen, seine kunsthistorische Würdigung mittels seiner künstlerischen und kulturhistorischen Kontextualisierung voranzutreiben. Das Jubiläumsjahr war Anstoß für gleich drei große Ausstellungen, die jeweils auch vielfältige Betrachtungen und Forschungen zu Slevogts Netzwerken ermöglichten: So die Ausstellung des Mainzer Landesmuseums Ein Tag am Meer. Slevogt, Liebermann \& Cassirer, ${ }^{5}$ so die fulminante Präsentation Slevogt und Frankreich im Saarlandmuseum Saarbrücken ${ }^{6}$ und so der retrospektive Abschluss des Jubiläums im Niedersächsischen Landesmuseum. ${ }^{7}$ Davon haben sowohl die öffentliche Sichtbarkeit der Kunst Slevogts als auch deren Erforschung enorm profitiert. Unser Band schließt daran an. Möge er für die Erforschung von Slevogts Netzwerken ein folgenreicher Start in die Zukunft sein. Er soll in weiteren Projekten fortgeführt werden, wozu sich im digitalen Zeitalter der Aufbau einer Forschungsinfrastruktur in Form einer Forschungsdatenbank anbietet, in der alle zusammengetragenen Informationen als digitale Zeugnisse und Forschungsdaten einer systematischen Analyse zugänglich sind. Dabei geht es darum, darauf zu achten, nicht wie in der Vergangenheit isolierte „Datenteiche“ anzulegen, sondern existierende oder noch zu bewerkstelligende Datenbanken miteinander zu verknüpfen, vulgo zu vernetzen, um sie zum „Datenozean der Zukunft" zu verschmelzen. ${ }^{8}$

Mein Dank gilt der Generaldirektion Kulturelles Erbe Rheinland-Pfalz, in Person ihres vormaligen Generaldirektors Dipl. Ing. Thomas Metz und seiner nunmehrigen Nachfolgerin im Amt, Dr. Heike Otto, die das Kolloquium und die nun vorliegende Publikation gefördert und finanziell möglich gemacht haben. Ebenso ist der Direktorin des Landesmuseums Mainz, Dr. Birgit Heide, für ihre immer verlässliche Unterstützung zu danken. Denjenigen Kolleginnen und Kollegen, die sich im Rahmen des Max Slevogt-Forschungszentrums engagieren, ist für die erfolgreiche gemeinsame Arbeit ein besonderer Dank auszusprechen: Dr. Eva Brachert und Dr. Karoline Feulner vom Landesmuseum Mainz sowie Dr. Armin Schlechter vom Landesbibliothekszentrum Rheinland-Pfalz/Pfälzische Landesbibliothek Speyer. Dank gilt auch unserer Kollegin Dr. Eva Wolf vom Saarlandmuseum in Saarbrücken, die mit zahlreichen Hilfestellungen und Auskünften unserem Kreis zuverlässig zur Seite steht. Wie immer war die so freundliche wie gut informierte Hilfe

5 Ein Tag am Meer. Slevogt, Liebermann \& Cassirer, hg. von Generaldirektion Kulturelles Erbe Rheinland-Pfalz, Kat. Ausst. Mainz, Landemuseum, München: Hirmer, 2018, S. 20-29.

6 Slevogt und Frankreich, hg. von Roland Mönig, Kat. Ausst. Saarbrücken, Saarlandmuseum, 2018.

7 Max Slevogt. Eine Retrospektive zum 150. Geburtstag, hg. von Thomas Andratschke, Kat. Ausst. Hannover, Niedersächsisches Landesmuseum, Petersberg: Imhof, 2018.

8 Torsten Schrade, „Im Datenozean. Chancen der nationalen Forschungsdatensammlung“, in: Frankfurter Allgemeine Zeitung, 29.11.2018. 
von Gernot Frankhäuser insbesondere mit Blick auf die Abbildungen von großem Wert. $\mathrm{Zu}$ deren Management hat meine studentische Hilfskraft Nicole Schkondin tatkräftig beigetragen. Die Mitarbeiter des De Gruyter-Verlags, namentlich Arielle Thürmel, Susanne Drexler und Jens Lindenhain, haben dem Buch ihr Auge und ihre Hand zur Verfügung gestellt und damit seine professionelle Produktion ermöglicht. Vor allem aber ist dem Netzwerk der Slevogtianer zu danken, jenen Kolleginnen und Kollegen, die zu Slevogt an Museen und Universitäten oder auch außerhalb davon forschen und zur Kenntnis und Verständnis seines Werkes beitragen. Zwischen ihnen hat sich in den letzten Jahren ein beständiger Kontakt entwickelt, der den großen Aufgaben, die im Hinblick auf diesen Künstlern noch zu stemmen sind, ein tragfähiges Fundament bietet. Einige von ihnen sind in diesem Band als Autoren vertreten. Ihre Texte sind Staffelstäbe, die aufgenommen werden wollen oder, um halbwegs im Bild zu bleiben, aufgewickelte Knäuel, aus denen Fäden herausgezogen und weitergesponnen werden können. 
